\title{
Magnetic resonance imaging as an alternative to computed tomography in select patients with traumatic brain injury: a retrospective comparison
}

\author{
Marie Roguski, MD, MPH, ${ }^{1}$ Brent Morel, BS, ${ }^{1,2}$ Megan Sweeney, ${ }^{3}$ Jordan Talan, BA, ${ }^{1,2}$ \\ Leslie Rideout, FNP, PhD, ${ }^{3}$ Ron I. Riesenburger, MD, ${ }^{1}$ Neel Madan, MD, ${ }^{4}$ and Steven Hwang, MD${ }^{1}$ \\ Departments of ${ }^{1}$ Neurosurgery, ${ }^{3}$ General Surgery, and ${ }^{4}$ Radiology, Tufts Medical Center; and ${ }^{2}$ Tufts University School of \\ Medicine, Boston, Massachusetts
}

\begin{abstract}
OBJECT Traumatic head injury (THI) is a highly prevalent condition in the United States, and concern regarding excess radiation-related cancer mortality has placed focus on limiting the use of $\mathrm{CT}$ in the evaluation of pediatric patients with THI. Given the success of rapid-acquisition MRI in the evaluation of ventriculoperitoneal shunt malfunction in pediatric patient populations, this study sought to evaluate the sensitivity of MRI in the setting of acute THI.

METHODS Medical records of 574 pediatric admissions for THI to a Level 1 trauma center over a 10-year period were retrospectively reviewed to identify patients who underwent both CT and MRI examinations of the head within a 5-day period. Thirty-five patients were found, and diagnostic images were available for 30 patients. De-identified images were reviewed by a neuroradiologist for presence of any injury, intracranial hemorrhage, diffuse axonal injury (DAI), and skull fracture. Radiology reports were used to calculate interrater reliability scores. Baseline demographics and concordance analysis was performed with Stata version 13.
\end{abstract}

RESULTS The mean age of the 30-patient cohort was $8.5 \pm 6.7$ years, and $63.3 \%$ were male. The mean Injury Severity Score was $13.7 \pm 9.2$, and the mean Glasgow Coma Scale score was $9 \pm 5.7$. Radiology reports noted 150 abnormal findings. CT scanning missed findings in 12 patients; the missed findings included DAI $(n=5)$, subarachnoid hemorrhage $(n=6)$, small subdural hematomas $(n=6)$, cerebral contusions $(n=3)$, and an encephalocele. The CT scan was negative in 3 patients whose subsequent MRI revealed findings. MRI missed findings in 13 patients; missed findings included skull fracture $(n=5)$, small subdural hematomas $(n=4)$, cerebral contusions $(n=3)$, subarachnoid hemorrhage $(n=3)$, and DAI $(n=1)$. MRI was negative in 1 patient whose preceding CT scan was read as positive for injury. Although MRI more frequently reported intracranial findings than $\mathrm{CT}$ scanning, there was no statistically significant difference between CT and MRI in the detection of any intracranial injury $(p=0.63)$, DAI $(p=0.22)$, or intracranial hemorrhage $(p=0.25)$. CT scanning tended to more frequently identify skull fractures than $\mathrm{MRI}(p=0.06)$.

CONCLUSIONS MRI may be as sensitive as CT scanning in the detection of THI, DAI, and intracranial hemorrhage, but missed skull fractures in 5 of 13 patients. MRI may be a useful alternative to $\mathrm{CT}$ scanning in select stable patients with mild THI who warrant neuroimaging by clinical decision rules.

http://thejns.org/doi/abs/10.3171/2014.10.PEDS14128

KEY WORDS pediatric; head injury; MRI; CT; trauma

$\mathrm{T}$ RAUMATIC head injury (THI) is a prevalent condition in the United States; an estimated 1.7 million people sustain THI in this country annually, and within that group, children aged 0 to 14 years account for 473,947 emergency room visits, 35,136 hospitalizations, and 2174 deaths annually. ${ }^{6} \mathrm{X}$-ray computed tomography (CT) has traditionally been the imaging modality of choice in the evaluation of patients with THI who require imaging, due largely to its ease and rapidity of acquisition. However, increasingly more attention is being focused on the potential cancer risks of CT-related radiation. ${ }^{4,13,17}$ Brenner et al. estimated the lifetime cancer mortality risk attributable to the radiation from a single CT scan of the head in a 1-yearold child to be $0.07 \%{ }^{4}$ Although this number is low, many

ABBREVIATIONS CT $=\mathrm{x}$-ray computed tomography; $\mathrm{DAI}=$ diffuse axonal injury; $\mathrm{MRI}=$ magnetic resonance imaging; $\mathrm{THI}=$ traumatic head injury . SUBMITTED March 12, 2014. ACCEPTED October 29, 2014.

INCLUDE WHEN CITING Published online February 20, 2015; DOI: 10.3171/2014.10.PEDS14128.

DISCLOSURE Dr. Madan reports direct stock ownership in Near Infrared Imaging. 
children with THI undergo more than $1 \mathrm{CT}$ scan, and this risk estimate is an order of magnitude higher than the lifetime cancer mortality risk estimate due to radiation related to a head CT in adults. In addition, this small risk to any given child may, in fact, translate to a large population-level risk, given that THI in children aged 0 to 14 years accounts for nearly half a million emergency room visits annually. ${ }^{6}$ Although the use of CT among children has decreased slightly over recent years with the creation and validation of clinical decision rules, such as the New Orleans Criteria (NOC), the Canadian CT Head Rule (CCHR), and the National Emergency X-Radiography Utilization Study II (NEXUS II) for CT scanning in pediatric patients with minor head injury, ${ }^{1,8,12,14,15}$ the utilization of CT in pediatric patients remains high and variable from center to center. ${ }^{3,9,10}$

The use of MRI of the brain in THI has historically and largely been limited to prognostication and to further diagnostic evaluation in patients with persistently poor neurological examinations. ${ }^{2,5,16,18,19}$ However, given the success of rapid-acquisition MRI in the evaluation of ventriculoperitoneal shunt malfunction in pediatric patient populations, ${ }^{11}$ we were interested in evaluating the feasibility of MRI as a replacement for or adjunct to CT in the radiological evaluation of select patients with mild THI. In doing so, we lay the groundwork for a future prospective study on the utility of MRI in pediatric patients presenting with THI.

\section{Methods}

Institutional review board approval was obtained for the study. Patients admitted to Tufts Medical Center, a Level 1 pediatric trauma center, with THI between January 2002 and December 2012 were identified and screened for eligibility; inclusion criteria included age less than 18 years and MRI of the brain obtained within 5 days of CT. Of 574 pediatric patients admitted with THI over the above time period, 35 patients met the inclusion criteria. Thirty patients had images available for review. In general, patients underwent further imaging with MRI for evaluation of continuing neurological impairment, for neurological deficits that were not explained by initial CT findings, or for prognostication. MRI studies included a localizer sequence, T2-weighted sequences, T2-FLAIR images, gradient-echo T2 images, and T1-weighted sequences. Medical records were reviewed for age, sex, Injury Severity Score, Glasgow Coma Scale score, and length of stay. A board-certified neuroradiologist (N.M.) reviewed randomly presented de-identified MR and CT images for presence of skull fracture, subdural or epidural hematoma, diffuse axonal injury (DAI), cerebral contusion, and subarachnoid or intraventricular hemorrhage. Radiology reports were also reviewed for the aforementioned pathologies to assess interrater reliability. Dedicated neuroradiologists generated all radiology reports at the time of the patient's admission.

Baseline demographic analysis and concordance analysis were performed using Stata version 13 (StataCorp). Results of descriptive analysis are reported as mean \pm standard deviation. Spearman rank correlation coefficients were generated separately for DAI, osseous injury, intracranial hemorrhage, and intracranial injury. McNemar's exact tests for correlated proportions were used to test the hypothesis of no difference between CT and MRI in detecting each category of injury. Sensitivity and specificity calculation used the modality with the highest rate of reporting as the gold standard. A single gold standard was avoided due to differential sensitivities of each modality to different types of injuries. In addition, interrater reliability between official reports and the blinded reviewer was assessed using percentage agreement, unweighted kappa coefficients, and Spearman correlation coefficients.

\section{Results}

The cohort comprised 30 patients ranging in age from 2 months to 18 years (Table 1 ). The mean age was $8.5 \pm 6.7$ years, and $63.3 \%$ of the patients were male. The mean Injury Severity Score was $13.7 \pm 9.2$, and the mean Glasgow Coma Scale score was $9 \pm 5$.7. All patients had MRI within 5 days of $\mathrm{CT}$ acquisition, but the mean number of days between CT and MRI was only $0.8 \pm 1.2$ days. The mean length of stay was $8.6 \pm 11$ days.

In the 60 imaging studies reviewed, 150 abnormal findings were noted. CT missed findings in 12 patients, including DAI $(n=5)$, subarachnoid hemorrhage $(n=6)$, small subdural hematomas $(n=6)$, cerebral contusions $(n=3)$, and an encephalocele. The CT scan of the head was negative in 3 patients whose subsequent MRI was positive for intracranial injury; the missed findings included a small subdural hematoma, 2 areas of traumatic subarachnoid hemorrhage, and a small area of FLAIR change of uncertain significance. Conversely, MRI missed findings in 13 patients. Missed findings included skull fracture $(\mathrm{n}=$ 5), small subdural hematomas $(n=4)$, cerebral contusions $(\mathrm{n}=3)$, subarachnoid hemorrhage $(\mathrm{n}=3)$, and DAI $(\mathrm{n}=$ 1). MRI was negative in 1 patient whose preceding $\mathrm{CT}$

TABLE 1. Baseline characteristics of 30 pediatric patients*

\begin{tabular}{cc}
\hline \multicolumn{1}{c}{ Variable } & Value \\
\hline Age (yrs) & \\
\hline Mean & $8.5 \pm 6.7$ \\
\hline Range & $0.2-18$ \\
\hline Percent male & $63.3 \%(19 / 30)$ \\
\hline Injury Severity Score & $13.7 \pm 9.2$ \\
\hline Mean & $1-35$ \\
\hline Range & $9 \pm 5.7$ \\
\hline Glasgow Coma Scale score & $3-15$ \\
\hline Mean & $0.8 \pm 1.2$ \\
\hline Range & $0-4$ \\
\hline Interval btwn CT \& MRI (days) & \\
\hline Mean & $8.6 \pm 11$ \\
\hline Range & $0-51$ \\
\hline Length of stay (days)
\end{tabular}

Means are reported \pm SD. 
scan was read as positive for injury; the missed finding in this patient was a focus of possible cerebral edema. Fig. 1 depicts 1 case in which CT missed a small subdural hematoma and 1 case in which MRI missed a right occipital nondisplaced skull fracture.

With respect to detection of any injury, although MRI of the head more frequently reported a traumatic injury than CT imaging of the head, this difference did not reach statistical significance in this cohort $(\mathrm{p}=0.63)$ (Table 2). The Spearman rank correlation coefficient of CT and MRI reporting of any injury was 0.63 . The sensitivity and specificity of CT scanning for detection of any injury were $87 \%$ and $85.7 \%$, respectively (Table 3 ).

MRI missed skull fractures in 5 patients; the Spearman rank correlation coefficient was 0.69 , indicating good validity of MRI in the assessment of skull fracture (Table 4). The difference between MRI and CT with respect to their ability to detect skull fractures was almost statistically significant $(\mathrm{p}=0.06)$. The sensitivity and specificity of MRI for skull fracture were $61.5 \%$ and $100 \%$, respectively (Table 3). Conversely, CT scanning missed DAI in 5 patients; the Spearman rank correlation coefficient of -0.08 indicates extremely poor validity of CT in the detection of DAI compared with MRI (Table 5). However, the 2 modalities were not significantly different in their abilities to detect DAI $(\mathrm{p}=0.22)$. The sensitivity and specificity of CT for detection of DAI were $0 \%$ and $96 \%$, respectively (Table 3).

Despite the above differences, good concordance was noted between MRI and CT for reporting of intracranial hemorrhage; the Spearman rank correlation coefficient of 0.80 indicates excellent concordance of MRI and CT (Table 6). Three CT scans of the head were reported as negative for intracranial hemorrhage when the corresponding MR image was reported as positive. In addition, despite the excellent concordance, CT scanning missed 6 small subdural hematomas and MRI missed 4 small subdural hematomas, but these patients also had other areas of intracranial hemorrhage. However, there was no statistically significant difference between CT and MRI in the detection of intracranial hemorrhage $(p=0.25)$. The sensitivity and specificity of CT scanning for detection of intracranial hemorrhage were $85.7 \%$ and $100 \%$, respectively (Table 3 ).

Interrater reliability scores between official reports and the blinded neuroradiologist's review were good to excellent for most of the measured indicators (Table 7) except for the diagnosis of DAI on CT and skull fracture on MRI. Agreement was noted to range from $80 \%$ to $93.3 \%$ for all categories, and most Kappa statistics were greater than 0.64. However, as mentioned earlier, 2 areas of low Kappa statistic values were noted. Regarding the detection of skull fracture on MRI, the neuroradiologist and official reports agreed with respect to $80 \%$ of the MR images, but this translated to a Kappa statistic of only 0.33 . The second area of low interrater reliability was in the detection of DAI on CT. The blinded neuroradiologist reported presence of DAI on only 1 of 30 CT scans; the official reports reported presence of DAI in 3 of 30 scans. Thus, although the 2 raters agreed in $86.7 \%$ of the scans, the Kappa statistic was -0.05 , reflecting extremely poor interrater reliability in the detection of DAI on CT scan.
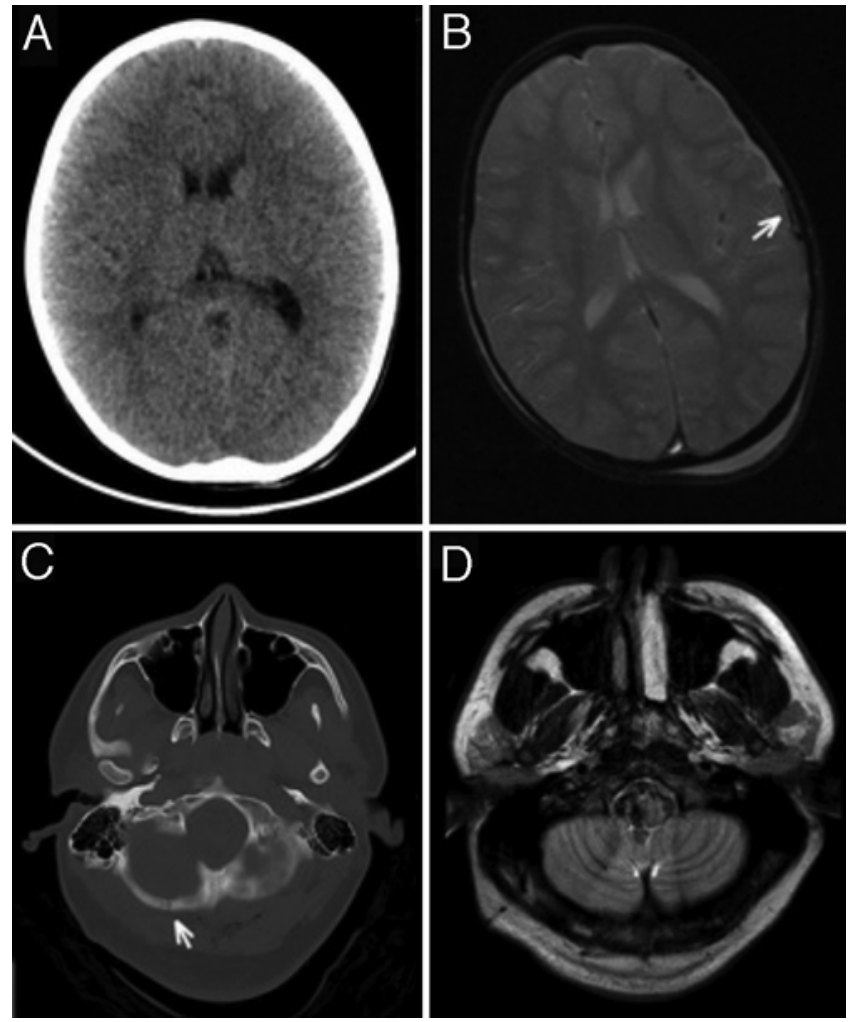

FIG. 1. Representative images from 2 cases in which one imaging modality missed an abnormality that was detected by the other. A and B: Axial CT image (A) and corresponding axial FLAIR MR image (B) showing a small subural hematoma that is not evident on the CT image. C and D: Axial CT image with bone windows $(C)$ showing a right occipital nondisplaced skull fracture (arrow) and corresponding axial T2-weighted MR image.

\section{Discussion}

The results of this study suggest that MRI may be a useful alternative to CT scanning in select patients. Although the study was limited due to the small sample size, MRI more commonly reported THI in general, DAI, and intracranial hemorrhage than the current primary neuroimaging modality used in the evaluation of patients with head trauma. Both imaging modalities missed findings in patients; however, most missed findings were subtle and clinically insignificant and were associated with other findings that were detected on the same scan. MRI reported no injury in 1 patient whose CT was positive for injury; the finding reported on CT was a small area that

TABLE 2. Table of results of whether injury was present on CT and MRI of the head*

\begin{tabular}{cccc}
\hline & & \multicolumn{2}{c}{ MRI: Injury Present? } \\
\cline { 3 - 4 } & & Yes & No \\
\hline CT: Injury & Yes & 20 & 1 \\
Present? & No & 3 & 6 \\
\hline
\end{tabular}

* Concordance analysis and McNemar's test results are provided. Spearman rank correlation coefficient $=0.67$. Exact McNemar's test, $p=0.63$. 
TABLE 3. Sensitivity and specificity of MRI and CT according to injury type*

\begin{tabular}{llll}
\hline \multicolumn{1}{c}{ Finding } & Prevalence $(95 \% \mathrm{Cl})$ & Sensitivity $(95 \% \mathrm{Cl})$ & \multicolumn{1}{c}{ Specificity $(95 \% \mathrm{Cl})$} \\
\hline Abnormal findings & $76.7 \%(57.7 \%-90.1 \%)$ & $87 \%(66.4 \%-97.2 \%)$ & $85.7 \%(42.1 \%-99.6 \%)$ \\
\hline DAl & $16.7 \%(5.6 \%-34.7 \%)$ & $0 \%(0 \%-52.2 \%)$ & $96 \%(79.6 \%-99.9 \%)$ \\
\hline Osseous injury & $43.3 \%(25.5 \%-62.6 \%)$ & $61.5 \%(31.6 \%-86.1 \%)$ & $100 \%(80.5 \%-100 \%)$ \\
\hline ICH & $70 \%(50.6 \%-85.3 \%)$ & $85.7 \%(63.7 \%-97 \%)$ & $100 \%(66.4 \%-100 \%)$ \\
\hline
\end{tabular}

$\mathrm{ICH}=$ intracranial hemorrhage.

* MRI is well established as the gold standard for soft-tissue imaging and thus is considered to be the gold standard for abnormal findings,

DAI, and intracranial hemorrhage. CT is well established as the gold standard for skeletal imaging and thus is considered to be the gold standard for skull fracture (osseous injury).

was suspicious for cerebral edema. On the other hand, CT reported no injury in 3 patients whose MRI was positive for injury. These injuries included a small subdural hematoma, 2 traumatic subarachnoid hemorrhages, and a small area of FLAIR change that may have correlated with a nonhemorrhagic contusion. Although these types of injuries would not necessarily distinguish between patients who require surgery and those who don't, they may determine the intensity of medical observation that is required. Overall, however, both modalities agreed on whether injury was present in nearly $87 \%$ of the studies. Despite these findings that strongly support the utility of MRI in the evaluation of pediatric THI, our analysis did not demonstrate a statistically significant difference $(\mathrm{p}=$ $0.63)$. Similar observations were noted in the detection of DAI and intracranial hemorrhage. Interestingly, although CT is commonly held to be superior at evaluating for intracranial hemorrhage, MRI detected hemorrhage in 3 patients with negative results on CT. On the other hand, CT did not detect any hemorrhage that was missed by MRI. Despite the lack of statistically significant difference between CT and MRI in detection of intracranial hemorrhage $(\mathrm{p}=0.25)$, these results strongly support the utility of MRI in the evaluation of intracranial hemorrhage.

To our knowledge, this is the first study to compare MRI and CT in the same individual patients in the setting of acute THI. Given the increasing focus on avoiding radiation and cancers attributable to $\mathrm{CT}$ and the comparability of MRI and CT in the detection of THI and intracranial hemorrhage noted above, MRI may be a useful adjunct to dose-reduced CT protocols in stable patients with mild THI who warrant neuroimaging by clinical decision protocols. However, there are several limiting factors regarding the implementation of MRI in the evaluation of pediatric THI. The sensitivity of MRI in the detection of skull fractures in this cohort was low, and MRI missed

TABLE 4. Table of results of whether osseous injury was present on CT and MRI of the head.*

\begin{tabular}{cccc}
\hline & & \multicolumn{2}{c}{ MRI: Osseous Injury Present? } \\
\cline { 2 - 3 } & & Yes & No \\
\hline CT: Osseous & Yes & 8 & 5 \\
\cline { 2 - 4 } Injury Present? & No & 0 & 17 \\
\hline
\end{tabular}

* Concordance analysis and McNemar's test results are provided. Spearman rank correlation coefficient $=0.69$. Exact McNemar's test, $p=0.06$.
5 of 13 skull fractures that were noted on CT scans. The low sensitivity of MRI for detection of skull fractures is a limitation of this modality, and perhaps patients with strong clinical evidence of skull fracture, such as a battle sign, bilateral orbital bruising, or significant scalp swelling, may be better evaluated with CT. Furthermore, the location of MR scanners in most hospitals as well as the limited observation possible within an MR scanner makes MRI unsuitable for many patients with signs and symptoms concerning for severe forms of THI. Lastly, the cost, time necessary for study acquisition, and commonly associated delays in care associated with acquisition of MR scans in the emergency room may limit the feasibility of MRI in the routine evaluation of head trauma. However, many large medical centers have succeeded in incorporating timely MRI in surgical decision making for patients in whom ventriculoperitoneal shunt malfunction is suspected; these patients are often at as high a risk of a negative outcome as many patients with mild to moderate THI, and the successful incorporation of MRI in their care may support the feasibility of MRI in the diagnostic evaluation of select pediatric patients with THI. In addition, regarding the poor sensitivity of MRI in the detection of skull fractures, all skull fractures in our cases were linear and nondisplaced, and none of the patients with skull fractures noted on CT scan required treatment for the skull fracture. Although accurate diagnosis is clearly important in patients with THI, it is notable that not all children with linear, nondisplaced skull fractures require hospital admission. In fact, in a retrospective, cross-sectional study of 3915 children with isolated skull fractures evaluated in emergency departments in US children's hospitals, only $78 \%$ of children were hospitalized, and, of those hospitalized, $85 \%$ were discharged within 1 day. ${ }^{7}$ The relatively benign natural history of uncomplicated, nondepressed skull fractures may ameliorate the poor sensitivity of MRI

TABLE 5. Table of results of whether DAI was present on CT and MRI of the head*

\begin{tabular}{rccc}
\hline & & \multicolumn{2}{c}{ MRI: DAI Present? } \\
\cline { 3 - 4 } & & Yes & No \\
\hline CT: DAI & Yes & 0 & 1 \\
Present? & No & 5 & 24 \\
\hline
\end{tabular}

* Concordance analysis and McNemar's test results are provided. Spearman rank correlation coefficient $=-0.08$. Exact McNemar's test, $p=0.22$. 
TABLE 6. Table of results of whether intracranial hemorrhage was present on CT and MRI of the head*

\begin{tabular}{cccc}
\hline & & \multicolumn{2}{c}{ MRl: ICH Present? } \\
\cline { 3 - 4 } & & Yes & No \\
\hline CT: ICH & Yes & 18 & 0 \\
Present? & No & 3 & 9 \\
\hline
\end{tabular}

* Concordance analysis and McNemar's test results are provided. Spearman rank correlation coefficient $=0.80$. Exact McNemar's test, $p=0.25$.

in the detection of skull fractures, especially if its implementation succeeds in reducing diagnostic radiation exposure in pediatric patients.

Previous studies that have evaluated MRI in trauma patients have focused on prognostication. ${ }^{5,16,18,19}$ Most MRI examinations performed in previously published studies were performed several weeks after the injury, and the sensitivity of MRI for intracranial injury may be limited by the long duration between imaging studies. The role of MRI in the present study was similar to that in previous studies: to evaluate continuing neurological impairment and neurological deficits that were not explained by initial CT findings, and to prognosticate. However, the mean time from CT scanning to MRI in this study was only 0.97 days, and in more than $75 \%$ of our cases, MR images were obtained within 3 days. The short duration between studies of these 2 modalities is a strength of this study, because the likelihood of substantial change between imaging studies is lower with a shorter elapsed time.

The study has a few limitations. First, as mentioned previously, all patients were admitted to the hospital with THI, and their inpatient care required both CT and MRI. MRI of the brain is not routinely obtained in the care of pediatric patients with THI. MRI in this cohort was often performed in THI patients in whom CT findings did not fully explain the severity of neurological impairments or for prognostication. This is supported by the relatively high prevalence of DAI in this cohort of $16.7 \%$. The selection of patients admitted to the hospital with THI and whose CT images may have only very subtle findings introduces bias that may skew the estimates in the favor of MRI. Un- fortunately, due to the retrospective nature of this study, we are unable to further clarify the indications for further imaging with MR, and, thus, although we acknowledge a significant selection bias by selecting patients with both types of imaging, it is difficult to specify the magnitude of this bias and its effect on our results and conclusions.

Concordantly, the patients in this series were deemed medically and neurologically stable for travel to MRI, and many of the findings were subtle, albeit detectable; it is logical to assume that MRI would be able to detect larger, less subtle findings as well. Thus, the strength of evidence suggests that MRI may be a useful modality to follow imaging findings that are found on CT. However, the study is retrospective and involves a relatively small number of patients. Because of these methodological limitations, we are unable to provide an algorithm regarding the optimal use of MRI in pediatric head trauma or offer suggestions about its use. Further prospective and randomized studies in patients with minor THI are needed to confirm whether MRI is a feasible alternative diagnostic modality. The patients in this study underwent full pediatric MRI of the brain with multiple sequences; further study is needed to determine which sequences most reliably identify intracranial injury and whether rapid acquisition sequences could be reliably integrated into a protocol. Despite these limitations, this is the first study to assess the sensitivity of MRI in the acute THI setting.

The study's limitations are real and limit our ability to make strong inferences or recommendations regarding what role MRI should serve in the evaluation and management of pediatric patients with THI; however, it serves as a feasibility study and as the basis for a future prospective pilot study of the role of MRI. The results provide some limited evidence of a substantial potential for radiation sparing in the vulnerable pediatric population and, thus, preliminary consideration of the role of MRI in pediatric THI may help avoid future CT-related excess cancer cases.

\section{Conclusions}

MRI may be a useful alternative to CT scanning in select patients with mild THI who warrant neuroimaging by clinical decision rules. MRI identified abnormalities such

TABLE 7. Inter-reader reliability scores between the blinded neuroradiology reviewer and official neuroradiology reports

\begin{tabular}{cllr}
\hline \multicolumn{1}{c}{ Variable } & Agreement & Kappa $(95 \% \mathrm{Cl})$ & Spearman $\rho$ \\
\hline MRI & & & 0.74 \\
\hline Injury present? & $90 \%$ & $0.73(0.45-1)$ & 0.44 \\
\hline Osseous injury present? & $80 \%$ & $0.33(-0.03$ to 0.68$)$ & 0.68 \\
\hline DAl present? & $86.7 \%$ & $0.64(0.33-0.95)$ & 0.80 \\
\hline ICH present? & $90 \%$ & $0.78(0.56-1)$ & 0.80 \\
\hline CT & & & 0.73 \\
\hline Injury present? & $90 \%$ & $0.78(0.56-1)$ & -0.06 \\
\hline Osseous injury present? & $86.7 \%$ & $0.72(0.47-0.97)$ & 0.86 \\
\hline DAl present? & $86.7 \%$ & $-0.05(-0.16$ to 0.05$)$ & $0.86(0.68-1)$ \\
\hline ICH present? & $93.3 \%$ & & \\
\hline
\end{tabular}


as intracranial hemorrhage and DAI more frequently than CT scanning, but had poor sensitivity in the evaluation of skull fractures. Further prospective study is needed to further clarify what role, if any, MRI should have in the management of pediatric patients with THI.

\section{References}

1. Atabaki SM, Stiell IG, Bazarian JJ, Sadow KE, Vu TT, Camarca MA, et al: A clinical decision rule for cranial computed tomography in minor pediatric head trauma. Arch Pediatr Adolesc Med 162:439-445, 2008

2. Beauchamp MH, Ditchfield M, Babl FE, Kean M, Catroppa $\mathrm{C}$, Yeates KO, et al: Detecting traumatic brain lesions in children: CT versus MRI versus susceptibility weighted imaging (SWI). J Neurotrauma 28:915-927, 2011

3. Berdahl CT, Vermeulen MJ, Larson DB, Schull MJ: Emergency department computed tomography utilization in the United States and Canada. Ann Emerg Med 62:486-494.e3, 2013

4. Brenner D, Elliston C, Hall E, Berdon W: Estimated risks of radiation-induced fatal cancer from pediatric CT. AJR Am J Roentgenol 176:289-296, 2001

5. Chelly H, Chaari A, Daoud E, Dammak H, Medhioub F, Mnif J, et al: Diffuse axonal injury in patients with head injuries: an epidemiologic and prognosis study of 124 cases. J Trauma 71:838-846, 2011

6. Faul M, Xu L, Wald MM, Coronado VG: Traumatic Brain Injury in the United States: Emergency Department Visits, Hospitalizations and Deaths 2002-2006. Atlanta, Centers for Disease Control and Prevention, 2010 (http://www. cdc.gov/traumaticbraininjury/pdf/blue_book.pdf) [Accessed January 7, 2015]

7. Hennelly KE, Mannix R, Nigrovic LE, Lee LK, Thompson $\mathrm{KM}$, Monuteaux MC, et al: Pediatric traumatic brain injury and radiation risks: a clinical decision analysis. J Pediatr 162:392-397, 2013

8. Kuppermann N, Holmes JF, Dayan PS, Hoyle JD Jr, Atabaki SM, Holubkov R, et al: Identification of children at very low risk of clinically-important brain injuries after head trauma: a prospective cohort study. Lancet 374:1160-1170, 2009

9. Larson DB, Johnson LW, Schnell BM, Goske MJ, Salisbury SR, Forman HP: Rising use of CT in child visits to the emergency department in the United States, 1995-2008. Radiology 259:793-801, 2011

10. Miglioretti DL, Johnson E, Williams A, Greenlee RT, Weinmann S, Solberg LI, et al: The use of computed tomography in pediatrics and the associated radiation exposure and estimated cancer risk. JAMA Pediatr 167:700-707, 2013

11. O’Neill BR, Pruthi S, Bains H, Robison R, Weir K, Ojemann $\mathrm{J}$, et al: Rapid sequence magnetic resonance imaging in the assessment of children with hydrocephalus. World Neurosurg 80:e307-e312, 2013

12. Osmond MH, Klassen TP, Wells GA, Correll R, Jarvis A, Joubert G, et al: CATCH: a clinical decision rule for the use of computed tomography in children with minor head injury. CMAJ 182:341-348, 2010
13. Pearce MS, Salotti JA, Little MP, McHugh K, Lee C, Kim $\mathrm{KP}$, et al: Radiation exposure from CT scans in childhood and subsequent risk of leukaemia and brain tumours: a retrospective cohort study. Lancet 380:499-505, 2012

14. Schachar JL, Zampolin RL, Miller TS, Farinhas JM, Freeman K, Taragin BH: External validation of the New Orleans Criteria (NOC), the Canadian CT Head Rule (CCHR) and the National Emergency X-Radiography Utilization Study II (NEXUS II) for CT scanning in pediatric patients with minor head injury in a non-trauma center. Pediatr Radiol 41:971979, 2011

15. Schonfeld D, Bressan S, Da Dalt L, Henien MN, Winnett JA, Nigrovic LE: Pediatric Emergency Care Applied Research Network head injury clinical prediction rules are reliable in practice. Arch Dis Child 99:427-431, 2014

16. Sigmund GA, Tong KA, Nickerson JP, Wall CJ, Oyoyo U, Ashwal S: Multimodality comparison of neuroimaging in pediatric traumatic brain injury. Pediatr Neurol 36:217-226, 2007

17. Smith-Bindman R, Lipson J, Marcus R, Kim KP, Mahesh M, Gould R, et al: Radiation dose associated with common computed tomography examinations and the associated lifetime attributable risk of cancer. Arch Intern Med 169:20782086, 2009

18. van der Naalt J, Hew JM, van Zomeren AH, Sluiter WJ, Minderhoud JM: Computed tomography and magnetic resonance imaging in mild to moderate head injury: early and late imaging related to outcome. Ann Neurol 46:70-78, 1999

19. Yuh EL, Mukherjee P, Lingsma HF, Yue JK, Ferguson AR, Gordon WA, et al: Magnetic resonance imaging improves 3 -month outcome prediction in mild traumatic brain injury. Ann Neurol 73:224-235, 2013

\section{Author Contributions}

Conception and design: Hwang, Roguski, Talan, Rideout, Riesenburger. Acquisition of data: Hwang, Roguski, Morel, Sweeney, Talan, Rideout, Riesenburger. Analysis and interpretation of data: all authors. Drafting the article: Hwang, Roguski, Morel, Rideout, Riesenburger, Madan. Critically revising the article: Hwang, Roguski, Rideout, Riesenburger, Madan. Reviewed submitted version of manuscript: Hwang, Roguski, Rideout, Riesenburger, Madan. Statistical analysis: Roguski, Riesenburger. Administrative/technical/material support: Hwang, Riesenburger. Study supervision: Hwang, Riesenburger.

\section{Supplemental Information \\ Previous Presentation}

Portions of this work were presented in e-poster format at the American Association of Neurosurgeons/Congress of Neurological Surgeons Joint Pediatric Section annual meeting in Toronto, Canada, on December 3-6, 2013.

\section{Correspondence}

Steven Hwang, Department of Neurosurgery, Tufts Medical Center, Proger 7, 800 Washington St., Boston, MA 02111. email: shwang@tuftsmedicalcenter.org. 